\title{
Recent results from the Pierre Auger Observatory
}

\author{
Sergio Petrera ${ }^{1,2, *}$ for the Pierre Auger Collaboration ${ }^{3, * *}$ \\ ${ }^{1}$ Gran Sasso Science Institute (GSSI), L'Aquila, Italy \\ ${ }^{2}$ INFN Laboratori Nazionali del Gran Sasso, Assergi (L'Aquila), Italy \\ ${ }^{3}$ Observatorio Pierre Auger, Av. San Martín Norte 304, 5613 Malargüe, Argentina \\ Full author list: http://www.auger.org/archive/authors_2018_08.html
}

\begin{abstract}
In this paper some recent results from the Pierre Auger Collaboration are presented. These are the measurement of the energy spectrum of cosmic rays over a wide range of energies $\left(10^{17.5}\right.$ to above $\left.10^{20} \mathrm{eV}\right)$, studies of the cosmic-ray mass composition with the fluorescence and surface detector of the Observatory, the observation of a large-scale anisotropy in the arrival direction of cosmic rays above $8 \times 10^{18} \mathrm{eV}$ and indications of anisotropy at intermediate angular scales above $4 \times 10^{19} \mathrm{eV}$. The astrophysical implications of the spectrum and composition results are also discussed. Finally the progress of the upgrade of the Observatory, AugerPrime is presented.
\end{abstract}

\section{Introduction}

The Pierre Auger Observatory [1] is the largest facility to detect cosmic rays built so far. It is located in the province of Mendoza, Argentina and has been in operation since 2004. Cosmic rays are studied by combining a Surface Detector (SD) and a Fluorescence Detector (FD) to measure extensive air showers. The SD consists of 1600 water-Cherenkov detectors on a $1500 \mathrm{~m}$ triangular grid (SD-1500) over an area of about $3000 \mathrm{~km}^{2}$, and of an additional 61 detectors covering $23.5 \mathrm{~km}^{2}$ on a $750 \mathrm{~m}$ grid (SD-750 or 'infill' array). The 24 fluorescence telescopes grouped in 4 FD buildings are located on the boundary of the observatory to overlook the whole atmospheric volume above the surface array. Three additional telescopes pointing at higher elevations (HEAT) are located near one of the FD sites (Coihueco) to detect lower energy showers. An array of radio antennas, Auger Engineering Radio Array (AERA) [1, 2], complements the data with the detection of the shower radiation in the hundred $\mathrm{MHz}$ region.

The design of the observatory has been conceived to exploit the "hybrid" concept, the simultaneneous detection of air showers by the surface array and fluorescence telescopes. The apparatus collects shower events of different classes depending on the on-time (generally called duty cycle) of the different detector components: the surface array is able to collect showers at any time, whereas the fluorescence detectors can operate only during clear moonless nights $(\approx 15 \%$ duty cycle $)$. Taking into account geometry and quality cuts applied at the event reconstruction level, the common data-set is only a few percent. Therefore only a small part of the SD showers are actually reconstructed by the FD. Nonetheless this sub-sample (the

\footnotetext{
*e-mail: sergio.petrera@aquila.infn.it

**e-mail: auger_spokespersons@fnal.gov
}

hybrid data-set) is very valuable, including events having both the footprint of the shower at ground and the longitudinal profile measured. The hybrid approach has been a major breakthrough in the detection of UHECRs since the method allows one to have the same energy scale in the surface detectors and the fluorescence telescopes and to derive the energy spectra entirely data-driven and free of model-dependent assumptions about hadronic interactions in air showers

In this paper I summarize some recent results from the Pierre Auger Observatory. Other interesting outcomes on several aspects of cosmic ray and particle physics that are not included here can be found e.g. in [3, 4].

\section{Energy spectrum}

One of the main goals of the Pierre Auger Observatory is to measure the cosmic ray spectrum at its highest energy end with unprecedented precision. This is done exploiting all data collected by the observatory, divided into 4 different samples. The SD-1500 and SD-750 samples are made of 'vertical' events (zenith angle $\theta<60^{\circ}$ for the standard and $\theta<55^{\circ}$ for the infill array) observed by the standard SD array and the infill array respectively. The 'inclined' sample is measured with the standard SD array but covers different zenith angles $\left(60^{\circ}<\theta<80^{\circ}\right)$ where a different reconstruction is needed. Finally the hybrid sample is made of events observed by both SD and FD.

The analysis method to derive the spectra is entirely data-driven. For the surface detector data, we transform the measured shower sizes to a size estimator that is independent of zenith angle by using the method of constant intensities [5] for the 'vertical' data and templates of the footprint of the particle densities at ground for the 'inclined' data set [6]. These attenuation-corrected shower 

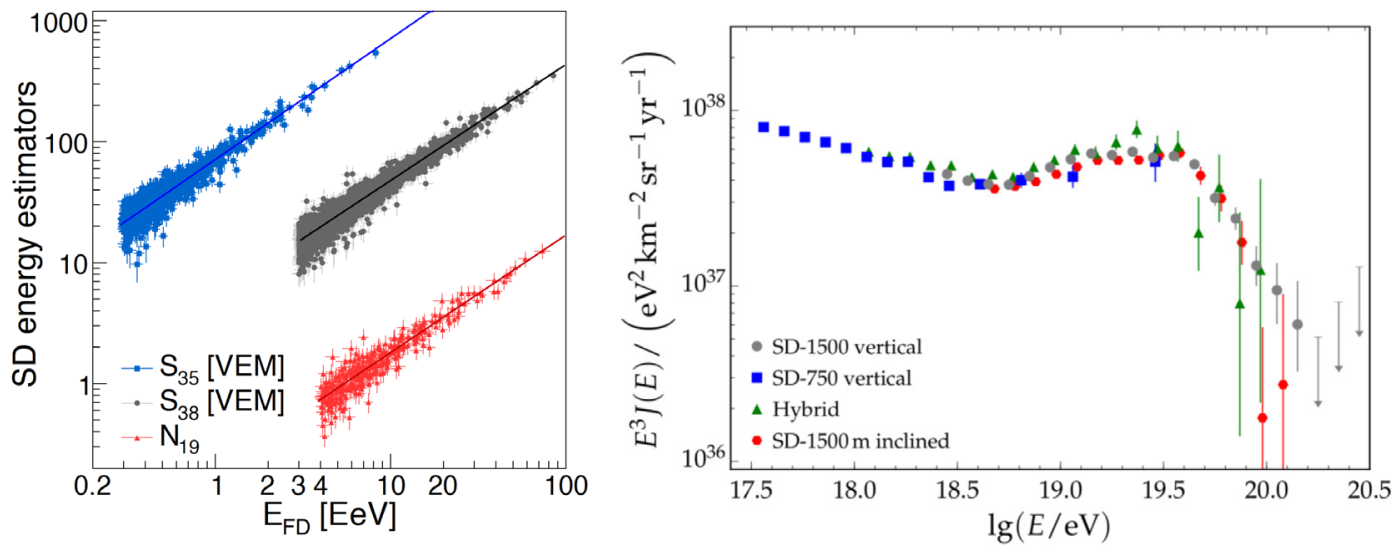

Figure 1. Left: Energy calibration of the surface detector. The shower size measured for 'vertical' events with the SD-1500 $\left(S_{38}\right)$ and SD-750 $\left(S_{35}\right)$ array and for inclined showers $\left(N_{19}\right)$ is shown as a function of the energy measured with the fluorescence telescopes $\left(E_{\mathrm{FD}}\right)$. Right: The energy spectra obtained with the four spectrum components. The systematic uncertainty on the energy scale, common to all of them, is $14 \%$

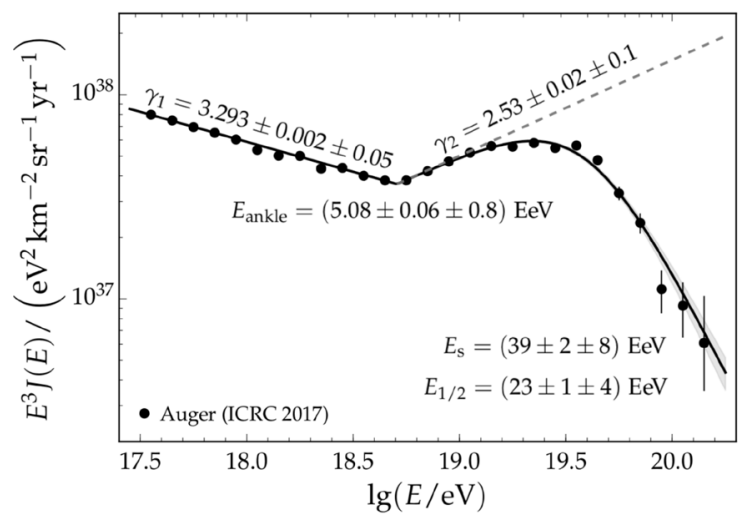

Figure 2. Combined energy spectrum. The line shows a fit to the spectrum with a broken power law and a suppression at ultrahigh energies. The gray dashed line indicates the same broken power law without suppression. The fitted spectral indices and energies of the break and suppression are superimposed together with their statistical and systematic uncertainties.

sizes are used as energy estimates after calibrating them with the calorimetric energy available for hybrid events as shown in the left panel of Fig. 1. Following this method all the spectrum components have the same energy scale. The overall systematic uncertainty of the energy scale remains at $14 \%[3,7,8]$.

All the spectra agree within the systematic uncertainties as shown in the right panel of Fig. 1, and are combined through a maximum likelihood fit in order to obtain the final spectrum. The combined energy spectrum [3, 7] is shown in Fig. 2 as presented at ICRC 2017. At the "ankle", observed at $E_{\text {ankle }}=5.08 \pm 0.06$ (stat.) \pm 0.8 (syst.) EeV, the spectral index hardens by $\Delta \gamma \sim-0.76$. A power law extension of the flux above the ankle is clearly excluded by data and we find a suppression en- ergy $^{1}$ of $E_{s}=39 \pm 2$ (stat.) \pm 8 (syst.) EeV with a spectral index softening of $\Delta \gamma_{s} \sim 2.5$. The energy at which the integral flux drops by a factor two below what would be expected without suppression is found to be $E_{1 / 2}=$ $23 \pm 1$ (stat) \pm 4 (syst) EeV. This value is considerably lower than $E_{1 / 2}=53 \mathrm{EeV}$ as predicted for the classical GZK scenario [9] where the suppression at ultrahigh energies is caused by the propagation of extra-galactic protons. However, the suppression of the spectrum can also be described by assuming a mixed composition at the sources or by the limiting acceleration energy at the sources rather than by the GZK-effect. Hence the energy spectrum alone remains ambiguous concerning astrophysical scenarios, which are better studied complementing the spectrum with other CR observables like mass composition and anistropy.

Comparing this energy spectrum with the one by Telescope Array one finds [10] that the ankle energies are consistent within the systematic uncertainties in the energy scale, but the discrepancy between the cut-off energies is not explained by systematics. An interesting question is whether the cutoff energy difference is due to a systematic bias or to astrophysics. A possible contribution to this difference in terms of declination dependence of the flux, as suggested by TA [10], has been investigated by Auger measuring the flux with the SD in different declination bands. No significant variation has been found that could account for the discrepancy between spectra measured from different hemispheres. The differences found between the flux measured in two separate declination bands, 'southern' ('northern'), corresponding to $\delta_{\mathrm{d}}<29.47^{\circ}\left(\delta_{\mathrm{d}}>29.47^{\circ}\right)$, are instead compatible with the variations expected from a dipolar modulation of the flux [11].

${ }^{1}$ We fitted the flux with a power law allowing for a break in the spectral index at $E_{\text {ankle }}$ and a suppression of the flux at ultrahigh energies $\propto\left[1+\left(E / E_{s}\right)^{\Delta \gamma_{s}}\right]^{-1}$ 

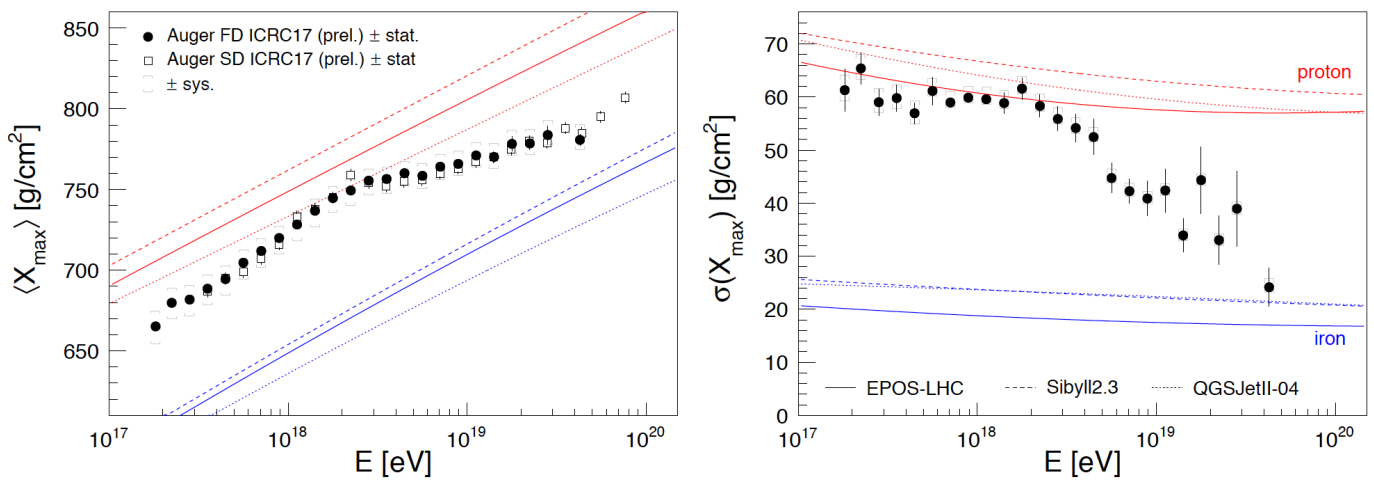

Figure 3. The mean (left) and the standard deviation (right) of the $X_{\max }$ distributions measured by Auger, as a function of energy compared to air-shower simulations for proton and iron primaries.

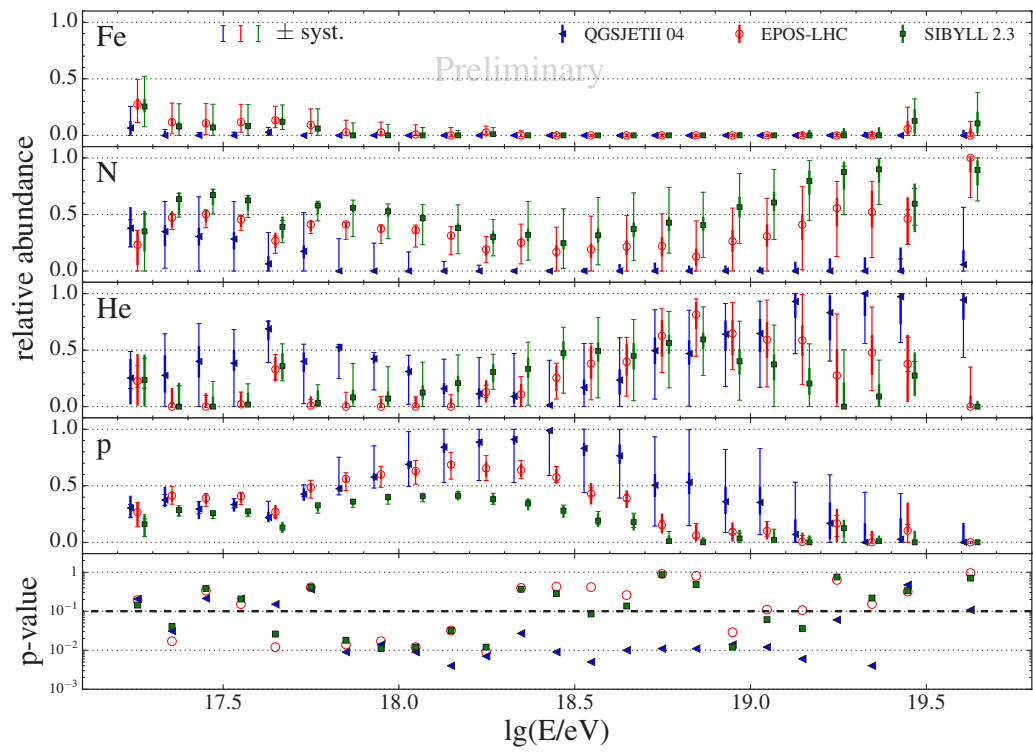

Figure 4. Results from a fit of the $X_{\max }$ distributions with a superposition of $\mathrm{H}, \mathrm{He}, \mathrm{N}$ and Fe induced air showers. The error bars indicate the statistics (smaller cap) and the systematic uncertainties (larger cap). The bottom panel indicates the goodness of the fits ( $p$-values).

\section{Mass composition}

Composition is addressed using the depth of the position of the maximum in the energy deposit of shower particles, $X_{\max }$, which is measured by the FD. In a simplistic picture, the sensitivity of $X_{\max }$ to mass composition relies on the fact that showers from heavier (lighter) nuclei develop higher (deeper) in the atmosphere and their profiles fluctuate less (more).

The measurements by Auger are robust for the accurate data selection and the statistical quality of the $X_{\max }$ distributions that are obtained. For the limited field of view of the telescopes, depending on the zenith angle and impact point of the shower, a fluorescence detector views a different range of $X_{\max }$. The Auger analysis adopts event selection and quality cuts that allow us to get rid of this bias and thus obtain unbiased $X_{\max }$ distributions. Cor- recting for detector resolution and acceptance, the first two moments of the distributions (mean and standard deviations) can be directly compared to air shower simulations. The Auger Collaboration has published $X_{\max }$ measurements for hybrid showers having energies above $10^{17.8}$ $\mathrm{eV}$ [12] and recently reported preliminary results extending these measurements down to $10^{17.2} \mathrm{eV}[3,13]$. Fig. 3 shows the latest data. In terms of average mass cosmic rays evolve towards a lighter composition between $10^{17.2}$ and $10^{18.3} \mathrm{eV}$, qualitatively corresponding to a transition from a heavy Galactic composition to a light extragalactic composition. At higher energies the trend is reversed and the average mass increases with energy.

The comparison of the $\left\langle X_{\max }\right\rangle$ energy dependence between Auger and TA is not immediate because different approaches are used to measure this observable by each 
experiment. In a report by the joint Auger-TA Working Group [14] methods to facilitate comparison of $\left\langle X_{\max }\right\rangle$ measurements are presented. Using these methods the Auger and TA composition results are shown to agree within the systematic uncertainties quoted by the two experiments. A paper by W. Hanlon in these proceedings also addresses this comparison study.

The statistics collected with the relatively low duty cycle of FD do not yet allow us to study the composition at energies where the flux suppression is observed. Using SD observables, it is however possible to cover also the highest energy range. In ref. [15] the time profiles of the signals recorded with the SD stations are employed to derive risetime. This observable depends on the distance of the shower maximum to ground and the relative amount of muons and electrons detected. The risetime-related variable $\Delta_{s}$ correlates with $X_{\max }$ and this allows to calibrate $\Delta_{s}$ to $\left\langle X_{\max }\right\rangle$. The energy evolution of $\left\langle X_{\max }\right\rangle$ from the SD is shown superimposed to the one from the FD measurements in the left panel of Fig. 3. As can be seen, the two measurements are in good agreement, as is to be expected due to the cross-calibration. At ultrahigh energies the superior statistics of the SD give two more data points. More and other analysis methods exploiting other SD-based observables will in future complement this method and provide a better understanding of this energy region.

The Auger $X_{\max }$ data (moments and distributions) enable a step further in the interpretation of mass composition studying the evolution with energy of the first two moments of $\ln A[3,12,13]$ and of the fractions of four mass groups $(\mathrm{H}, \mathrm{He}, \mathrm{N}$ and $\mathrm{Fe})$ from the fit of the $X_{\max }$ distributions $[3,13,16]$. The latest results are shown in Fig. 4. At the lowest energies, we find hints for a contribution $(25 \div 38 \%$ depending on models) from iron primaries that disappears rapidly with increasing energy. At high energies the composition is dominated by a different elemental group, starting from protons below the ankle and going through helium to nitrogen as the energy increases. This evolution occurs with limited mass mixing as a consequence of the small values of the $X_{\max }$ dispersion. The interpretation of $X_{\max }$ data depends on the hadronic interaction models. In particular QGSJetII-04 appears to be less consistent with data as can be seen in the lower panel of Fig. 4, where the probability of the fits is shown.

\section{Astrophysical implications from spectrum and composition data}

The results shown in the previous section show our current knowledge of the mass composition of UHECRs as they hit the Earth. In Sec. 2 I pointed out that despite the accurate measurement of the energy spectrum an interpretation in terms of sources is ambiguous. Instead using both spectrum and composition one can remove this degeneracy and infer information about the source scenario(s) which is(are) compatible with data. Several investigations have been done in recent years to interpret the UHECR spectrum and composition [17]. Most of these studies converge to scenarios with sources injecting hard spectra with
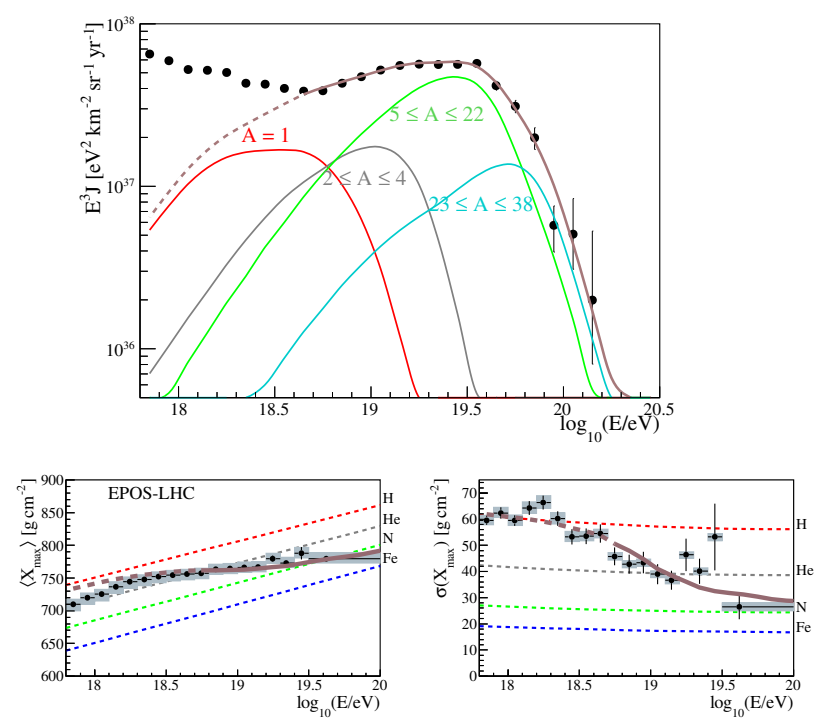

Figure 5. Top: energy spectrum obtained with the best-fit parameters for the SPG model (SimProp code, PBS photo-production cross-sections, Gilmore '12 EBL) [23]. Partial spectra are shown with different colors, total spectrum with brown. Full circles show the ICRC 2015 Auger spectrum. Bottom: average and standard deviation of the $X_{\max }$ for data [12] (full circles) and best-fit prediction using EPOS-LHC (brown). Pure H (red), He (grey), N (green) and $\mathrm{Fe}$ (blue) are shown as dashed lines. Only the energy range where the brown lines are solid is included in the fit.

low rigidity cutoff and mixed composition, even though simplifying assumptions are used as uniform source distributions and $1 \mathrm{D}$ cosmic ray propagation. These studies mainly address the description of the data above the ankle because lower energy data need additional hypotheses as new source populations or interactions of ejected cosmic rays in the radiation field surrounding the source $[18,19]$. All these results are amply model dependent [20]: besides the hadronic interaction models which describe the shower development in the atmosphere, the other model uncertainties come from the extra-galactic background light (EBL) radiation which cosmic rays cross in their propagation and the cross sections of photo-disintegration of nuclei interacting with background photons. These uncertainties are sizeable and mainly due to the lack of data [21].

The Auger Collaboration has published a comprehensive study about the astrophysical implications from the combined fit of spectrum and composition data [22], discussing in detail the effects of theoretical uncertainties on propagation and interactions in the atmosphere of UHECRs as well as the dependence of the fit parameters on the experimental systematic uncertainties. In this study we used a scenario in which the sources of UHECRs are of extragalactic origin and accelerate nuclei in electromagnetic processes with a rigidity-dependent maximum energy, $E_{\max }(Z)=E_{\max }(p) \times Z$, where $Z$ denotes the charge and $E_{\max }(p)$ is the maximum energy for protons. Within this scenario a good description of the shape of the measured energy spectrum as well as the energy evolution of the $X_{\max }$ distributions can be achieved if the sources accel- 


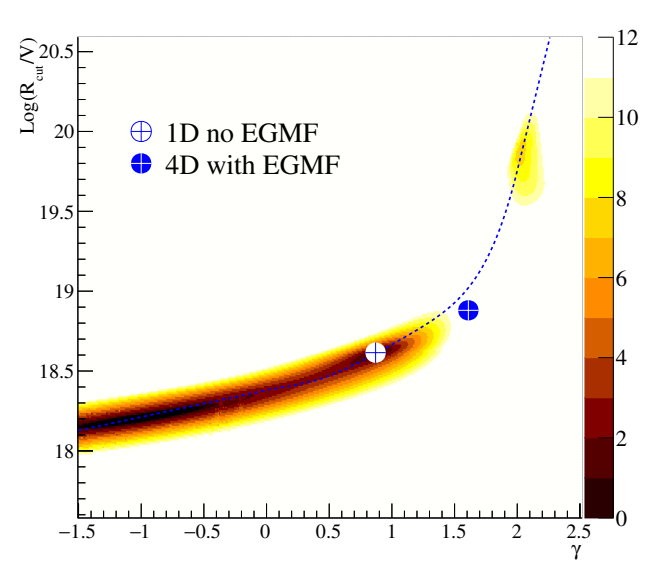

Figure 6. Pseudo standard deviation $\sqrt{D-D_{\min }}$ (where $D$ is the deviance) as function of $\gamma$ and $\log _{10}\left(R_{\text {cut }} / \mathrm{V}\right)$ for the $1 \mathrm{D}$ propagation fit [22]. The filled blue (white) marker shows the position of the minimum for $4 \mathrm{D}$ propagation with LSS sources and EGMF (1D propagation).

erate a primary nuclear mix consisting of $\mathrm{H}, \mathrm{He}, \mathrm{N}$ and $\mathrm{Si}$, if the primary spectrum follows a power law $\propto E^{-\gamma}$ with a spectral index $\gamma \approx 1$ and if the maximum energy of protons is about $10^{18.7} \mathrm{eV}$, as shown in Fig. 5. The mass composition at the sources is dominated by intermediate mass nuclei $(\mathrm{N}, \mathrm{Si})$. Using different hypotheses (i.e. hadronic interaction and EBL models, photo-disintegration cross sections) [23] source parameters can change considerably and for some of them well outside the statistical uncertainties of the fit with other assumptions. However the low spectral index and low rigidity solution is generally preferred.

In a more recent work $[3,24]$ the homogeneous distribution of sources assumed in our previous calculations was replaced by discrete sources distributed according to the model of the local large-scale structure from [25], with a source density of $10^{-4} \mathrm{Mpc}^{-3}$. Moreover, we studied the effects of the extragalactic magnetic field (EGMF) using the EGMF model proposed in [26], which describes a relatively strong magnetic field, together with reflective boundary conditions. To pursue this program the 4D mode of the Monte Carlo code CRPropa 3 [26] was used. The combined fit of spectrum and composition provides a good overall description of the data (similar to the one achieved with the simpler model). Comparing the best-fit parameters of the extended model with our previous results, we found that the details of the local large-scale structure are of minor importance for the derived parameters of the source spectra. Yet by including the diffusion in the EGMF in the calculation we derive a spectral index of $\gamma \sim 1.6$, i.e. significantly softer than the one obtained without magnetic fields. Fig. 6 shows the change of the spectral parameters from the 1D (uniform, no EGMF) to the 4D (LSS source distribution + EGMF). The presence of magnetic fields in intergalactic space should therefore be taken into account when interpreting cosmic ray data, especially if the field strength is relatively strong as assumed in this study.

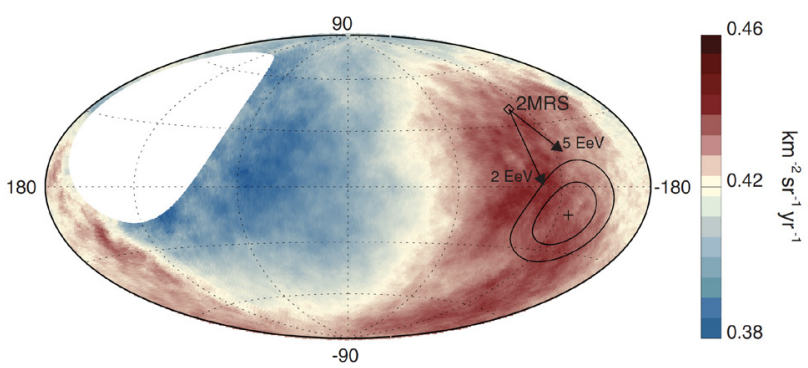

Figure 7. Sky map in galactic coordinates showing the cosmicray flux for $E \geq 8 \mathrm{EeV}$ smoothed with a $45^{\circ}$ top-hat function. The galactic center is at the origin. The cross indicates the measured dipole direction; the contours denote the $68 \%$ and $95 \%$ confidence level regions. The dipole in the 2MRS galaxy distribution is indicated. Arrows show the deflections expected for a particular model of the galactic magnetic field [28] on particles with $E / Z=5$ or $2 \mathrm{EeV}$.

\section{Anisotropy}

The Auger Collaboration has undertaken several anisotropy searches at different energy ranges and angular scales. These use several tools like harmonic analysis, auto-correlation, correlation with source catalogs, search for flux excesses in the visible sky and correlations with other experiments.

Among the most recent studies the observation of a large-scale anisotropy in the arrival directions of cosmic rays above $8 \times 10^{18} \mathrm{eV}$ [27] is indeed the most exciting. Two energy bins, $4 \mathrm{EeV}<E<8 \mathrm{EeV}$ and $E \geq 8 \mathrm{EeV}$, were analysed since the start of data taking (total exposure of $76,800 \mathrm{~km}^{2} \mathrm{sr} \mathrm{yr}$ ) with the Observatory measuring the amplitude of the first harmonic in right ascension. The right ascension anisotropy found in the two energy bins has amplitude $0.5_{-0.2}^{+0.6 \%}$ and $4.7_{-0.7}^{+0.8} \%$, respectively. The events in the lower energy bin follow an arrival distribution consistent with isotropy, but in the higher energy bin a significant anisotropy was found, with a $p$-value of $2.6 \times 10^{-8}$ under the isotropic null hypothesis. The three-dimensional dipole, obtained combining the first-harmonic analysis in right ascension with a similar one in the azimuthal angle, has a direction in galactic coordinates $(l, b)=\left(233^{\circ},-13^{\circ}\right)$ about $125^{\circ}$ away from the Galactic Centre hence indicating an extragalactic origin for these UHECR particles. The dipole anisotropy is detected at more than a $5.2 \sigma$ level of significance with an amplitude of $6.5_{-0.9}^{+1.3} \%$. A skymap of the intensity of cosmic rays arriving above $8 \mathrm{EeV}$ is shown in Fig. 7.

Some large scale anisotropy is expected because of the relative motion of cosmic rays with respect to the rest frame of background radiation [29] but the amplitude is expected to be below the percent level, well below what has been observed. Other studies have predicted larger anisotropies originating from an inhomogeneous distribution of sources, or that they arise from a dominant source. To illustrate this effect we considered the distribution of nearby galaxies, as mapped by the 2MASS redshift survey (2MRS) [30], which exhibits a dipolar structure. Fig. 7 
shows the direction toward the $2 \mathrm{MRS}$ dipole and the expected deflections caused by the galactic magnetic field. It is worth noting that the agreement between the directions of the UHECR and 2MRS dipoles is improved by adopting assumptions consistent with the observed charge composition and the deflections in the Galactic magnetic field.

An extension of the analysis of the large-scale anisotropy has been recently submitted [31]. Here both the dipolar and quadrupolar components are studied in the two energy ranges and further the bin above $8 \mathrm{EeV}$ is analysed by splitting it into three so as to explore how the amplitude and phase of the dipole changes with energy. The quadrupolar component is found to be not statistically significant. Instead we find that the amplitude of the dipole increases with energy above $4 \mathrm{EeV}$, as expected by predictions from models [32].

The search for flux excesses does not show statistically significant evidence of anisotropy [33, 34]. Yet remarkable flux excesses is observed at intermediate scales. The strongest departure from isotropy (post-trial significance $\sim 3.1 \sigma)$ is obtained for cosmic rays with $E>58$ $\mathrm{EeV}$ around the direction of Cen $\mathrm{A}$ ( $15^{\circ}$ radius). It is remarkable to recall that for cosmic ray events with energy $E>57 \mathrm{EeV}$, Telescope Array have observed in the northern sky a cluster of events (hotspot) [35], centered at $\alpha_{\mathrm{d}}=146.7^{\circ}, \delta_{\mathrm{d}}=43.2^{\circ}$, of about $20^{\circ}$ radius and with a calculated probability of appearing by chance in an isotropic cosmic-ray sky of $3.7 \times 10^{-4}(3.4 \sigma)$. It will be interesting to follow the evolution of these excesses with future data from both experiments.

A different type of analysis [36] has also been performed, based on the assumption that the UHECR flux is proportional to non-thermal electromagnetic flux. This analysis then takes into account the different flux of the single candidate sources. Two different candidate sources were taken into account: AGNs and starburst (starforming) galaxies (SBG). Active galaxies were extracted from the Fermi-LAT 2FHL Catalog [37], selecting only radioloud AGNs within a $250 \mathrm{Mpc}$ radius. A list of $17 \mathrm{bright}$ nearby candidates was obtained this way, and their integral $\gamma$-ray fluxes between $50 \mathrm{GeV}$ and $2 \mathrm{TeV}$ were used as a proxy for the UHECR flux. For SBGs [38], since only a few of them were observed directly in the $\gamma$-ray band, continuum emission at $1.4 \mathrm{GHz}$ was used as a proxy for the UHECR flux so that the 23 brightest nearby objects with a radio flux larger than $0.3 \mathrm{Jy}$ were selected. The attenuation of the intensity due to energy losses in the propagation to Earth is taken into account. The free parameters of this study are the threshold energy, the smearing angle and the fraction of anisotropic cosmic rays originating from the tested intensity model. The evolution of the test statistic (the likelihood ratio between model and isotropy) as a function of threshold energy is shown in Fig. 8. The TS is then maximized as a function of two free parameters in different energy threshold from 20 up to $80 \mathrm{EeV}$. In Figure 9, the TS as a function of the two free parameters is shown for the energy threshold where the maximum is found: $60 \mathrm{EeV}$ for the AGNs and $39 \mathrm{EeV}$ for the starburst galaxies. The best-fit parameters are found to be a smearing angle of $13^{\circ}$ and an anisotropic fraction of $10 \%$

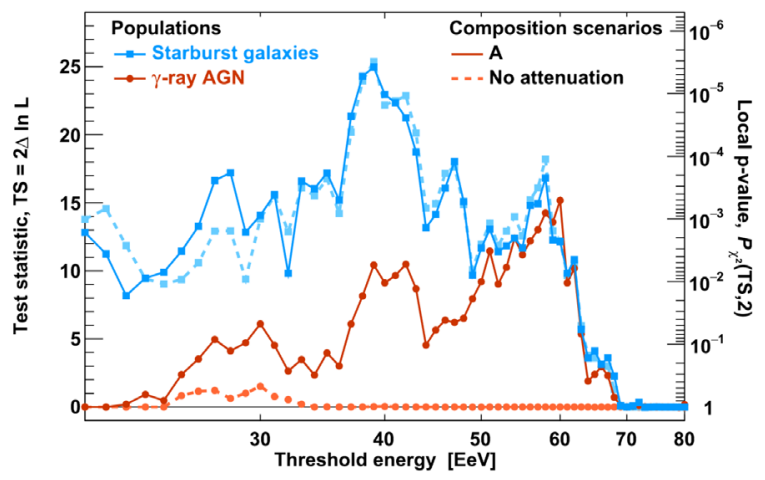

Figure 8. Test statistic (TS) scan over the threshold energy for SBGs and $\gamma$-AGNs. The continuous lines indicate the values of the test statistics obtained accounting for attenuation of the intensity due to energy losses while the dotted lines refer to the values without any attenuation.

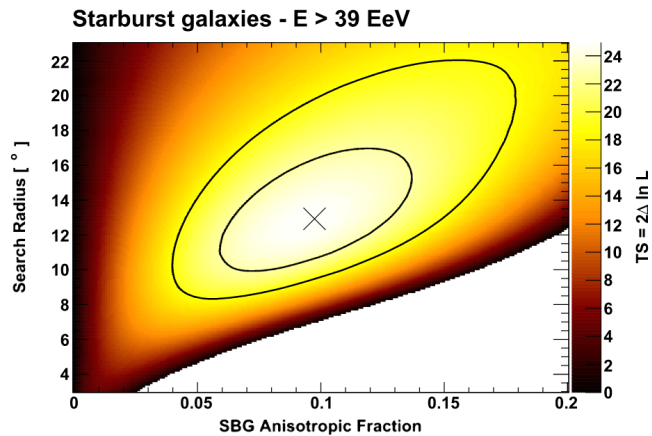

Active galactic nuclei - $\mathrm{E}>60 \mathrm{EeV}$

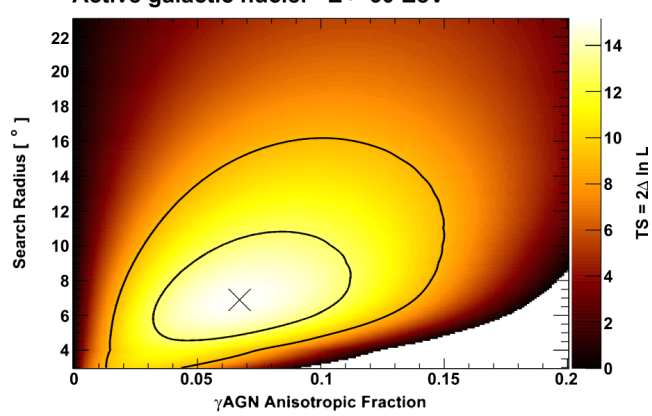

Figure 9. TS profile above $39 \mathrm{EeV}$ (top) and $60 \mathrm{EeV}$ (bottom) over the fit parameters for SBG and $\gamma \mathrm{AGN}$ models. The lines indicate the $1 \sigma-2 \sigma$ regions.

for the starburst-galaxies with a TS of 24.9, corresponding to a significance of $\sim 4 \sigma$, and $7^{\circ}$ and $7 \%$ for the $\gamma$-ray AGNs with a TS of 15.2 corresponding to a significance of $\sim 2.7 \sigma$. It is remarkable that while the significance for the AGNs is close to the one obtained in a previous analysis [33], a much higher significance is obtained with the newly tested starburst hypotesis. 


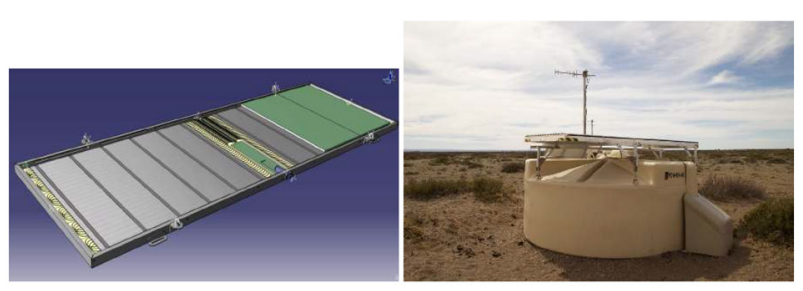

Figure 10. Left: the layout of the Surface Scintillator Detector (SSD). Right: One station of the AugerPrime Engineering Array.

\section{Future prospects: AugerPrime}

UHECR data provide several interesting outcomes on several aspects of cosmic ray physics as the ones presented in this work, but also others including photon and neutrino limits, multi-messengers studies and hadronic interactions at energies higher than LHC, not discussed here. Despite the progresses in our understanding it appears still difficult to build a consistent picture of the origin of UHECRs. To make further progress in this direction more accurate and extended information on the nature of the primaries is required: mass composition is currently unavailable above $40 \mathrm{EeV}$ due to the intrinsic duty cycle of the FD and the scarce accuracy of the composition sensitive methods based on the surface array data.

The AugerPrime upgrade [39] of the Pierre Auger Observatory has been specifically designed to improve mass composition in the whole energy range. Along the line of a hybrid design, each SD will be equipped with a top scintillator layer. Shower particles will be sampled by two detectors (scintillators and water-Cherenkov stations) having different responses to the muonic and electromagnetic components. The combined measurement allows to disentangle these two components and to provide an estimate of both, mass and energy of the shower, on an event-by-event basis. The detectors will be read out by new electronics with a faster and more accurate sampling of the signal. An extra small photomultiplier installed in each WCD will extend the current dynamic range to more than 32 times the largest signals currently measured. The upgraded array will provide data with no duty cycle limitation and then the access to the highest energies will be made possible. This setup is complemented by an underground muon detector AMIGA [40] in the current infill array. Finally we plan to increase the current FD duty cycle by $\sim 50 \%$ extending the operational mode to periods with a higher night sky background.

The AugerPrime Engineering Array (EA) [3, 41] consisting of 12 AugerPrime detector stations is in operation since 2016. The layout of the Surface Scintillator Detector (SSD) stations is shown in Fig. 10 together with the picture of one upgraded station of the EA. With this setup we have verified the basic functionality of the detector design, the linearity of the scintillator signal, the calibration procedures and operational stability. The upgraded stations produce signals that are in good agreement with expecta-
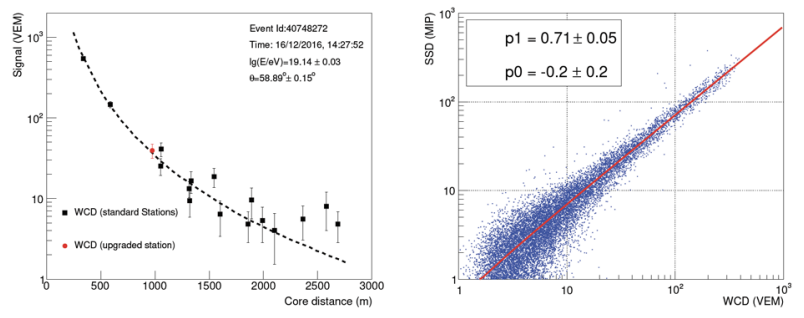

Figure 11. Left: one event reconstructed with the regular $1500 \mathrm{~m}$ array in close proximity to the EA. The reconstructed signals in the EA are compared with the LDF of the event. Right: correlation of the signals of the SSD and the WCD. Both signals have been calibrated.

tions as shown in Fig. 11 for one event reconstructed from EA data.

The construction of AugerPrime is expected to be finished by 2019 and it will take data until 2025.

\section{Acknowledgements}

The successful installation, commissioning, and operation of the Pierre Auger Observatory would not have been possible without the strong commitment and effort from the technical and administrative staff in Malargüe, and the financial support from a number of funding agencies in the participating countries, listed at https://www.auger.org/index.php/about-us/funding-agencies.

\section{References}

[1] A. Aab et al. [Pierre Auger Collaboration], Nucl. Instrum. Meth. A 798, 172 (2015).

[2] P. Abreu et al. [Pierre Auger Collaboration], JINST 7 (2012) P10011.

[3] D. Veberič [Pierre Auger Collaboration], arXiv:1708.06592 [astro-ph.HE].

[4] M. Unger [Pierre Auger Collaboration], PoS ICRC 2017 (2018) 1102.

[5] J. Hersil, I. Escobar, D. Scott, G. Clark and S. Olbert, Phys. Rev. Lett. 6 (1961) 22.

[6] A. Aab et al. [Pierre Auger Collaboration], JCAP 1408 (2014) no.08, 019.

[7] F. Fenu [Pierre Auger Collaboration], PoS ICRC 2017 (2018) 486.

[8] V. Verzi [Pierre Auger Collaboration], Proc. 33th ICRC 2013, Rio de Janeiro, Brazil [arXiv:1307.5059].

[9] V. Berezinsky, A. Z. Gazizov and S. I. Grigorieva, Phys. Rev. D 74 (2006) 043005.

[10] T. AbuZayyad et al., JPS Conf. Proc. 19 (2018) 011003.

[11] I. Valino [Pierre Auger Collaboration], PoS ICRC 2015 (2016) 271.

[12] A. Aab et al. [Pierre Auger Collaboration], Phys. Rev. D 90 (2014) no.12, 122005.

[13] J. Bellido [Pierre Auger Collaboration], PoS ICRC 2017 (2018) 490. 
[14] W. Hanlon et al., JPS Conf. Proc. 19 (2018) 011013.

[15] A. Aab et al. [Pierre Auger Collaboration], Phys. Rev. D 96 (2017) no.12, 122003.

[16] A. Aab et al. [Pierre Auger Collaboration], Phys. Rev. D 90 (2014) no.12, 122006.

[17] References can be found e.g. in R. Aloisio, P. Blasi, I. De Mitri and S. Petrera, Multiple Messengers and Challenges in Astroparticle Physics (Springer, 2018) 195 and arXiv:1707.06147 [astro-ph.HE].

[18] M. Unger, G. R. Farrar and L. A. Anchordoqui, Phys. Rev. D 92 (2015) no.12, 123001.

[19] N. Globus, D. Allard and E. Parizot, Phys. Rev. D 92 (2015) no.2, 021302.

[20] R. Alves Batista, D. Boncioli, A. di Matteo, A. van Vliet and D. Walz, JCAP 1510 (2015) no.10, 063.

[21] For photo-disintegration uncertainties see e.g. D. Boncioli, A. Fedynitch and W. Winter, Sci. Rep. 7 (2017) no.1, 4882.

[22] A. Aab et al. [Pierre Auger Collaboration], JCAP 1704 (2017) no.04, 038, Erratum: [JCAP 1803 (2018) no.03, E02].

[23] References about the models can be found in [22].

[24] D. Wittkowski [Pierre Auger Collaboration], PoS ICRC 2017 (2018) 563.

[25] K. Dolag, D. Grasso, V. Springel and I. Tkachev, JCAP 0501 (2005) 009.

[26] R. Alves Batista et al., JCAP 1605 (2016) no.05, 038.

[27] A. Aab et al. [Pierre Auger Collaboration], Science 357 (2017) no.6537, 1266.
[28] R. Jansson and G. R. Farrar, Astrophys. J. 757 (2012) 14.

[29] M. Kachelriess and P. D. Serpico, Phys. Lett. B 640 (2006) 225.

[30] P. Erdogdu et al., MNRAS 368 (2006) 1515.

[31] A. Aab et al. [Pierre Auger Collaboration], Submitted to: Astrophys.J. [arXiv:1808.03579 [astro-ph.HE]].

[32] D. Harari, S. Mollerach and E. Roulet, Phys. Rev. D 92 (2015) no.6, 063014.

[33] A. Aab et al. [Pierre Auger Collaboration], Astrophys. J. 804 (2015) no.1, 15.

[34] G. U. Giaccari [Pierre Auger Collaboration], PoS ICRC 2017 (2018) 483.

[35] R. U. Abbasi et al. [Telescope Array Collaboration], Astrophys. J. 790 (2014) L21

[36] A. Aab et al. [Pierre Auger Collaboration], Astrophys. J. 853 (2018) no.2, L29.

[37] M. Ackermann et al. [Fermi-LAT Collaboration], Astrophys. J. Suppl. 222 (2016) no.1, 5.

[38] Y. Gao and P. M. Solomon, Astrophys. J. Suppl. 152 (2004) 63.

[39] A. Aab et al. [Pierre Auger Collaboration], arXiv:1604.03637 [astro-ph.IM].

[40] A. Aab et al. [Pierre Auger Collaboration], JINST 11 (2016) no.02, P02012.

[41] D. Martello [Pierre Auger Collaboration], PoS ICRC 2017 (2018) 383. 